CUBO A Mathematical Journal

Vol.19, No 02, (11-31). June 2017

\title{
On the hypercontractive property of the Dunkl-Ornstein-Uhlenbeck semigroup
}

\author{
IRIS A. LÓPEz 1 \\ Departamento de Matemáticas Puras y Aplicadas, \\ Universidad Simón Bolivar, \\ Aptdo 89000. Caracas 1080-A. Venezuela. \\ iathamaica@usb.ve
}

\begin{abstract}
The aim of this paper is to prove the hypercontractive propertie of the Dunkl-OrnsteinUhlenbeck semigroup, $\left\{e^{\left(\mathrm{tL}_{k}\right)}\right\}_{\mathrm{t} \geq 0}$. To this end, we prove that the Dunkl-OrnsteinUhlenbeck differential operator $L_{k}$ with $k \geq 0$ and associated to the $\mathbb{Z}_{2}^{d}$ group, satisfies a curvature-dimension inequality, to be precise, a $\mathrm{C}(\rho, \infty)$-inequality, with $0 \leq \rho \leq 1$. As an application of this fact, we get a version of Meyer's multipliers theorem and by means of this theorem and fractional derivatives, we obtain a characterization of Dunkl-potential spaces.
\end{abstract}

\section{RESUMEN}

El objetivo de este artículo es demostrar la propiedad hipercontractiva del semigrupo de Dunkl-Ornstein-Uhlenbeck, $\left\{e^{\left(\mathrm{tL}_{k}\right)}\right\}_{\mathrm{t} \geq 0}$. Para lograr esto, probamos que el operador diferencial de Dunkl-Ornstein-Uhlenbeck $L_{k}$ con $k \geq 0$ y asociado al grupo $\mathbb{Z}_{2}^{\mathrm{d}}$, satisface una desigualdad de curvatura-dimensión, para ser precisos, una $\mathrm{C}(\rho, \infty)$-desigualdad, con $0 \leq \rho \leq 1$. Como una aplicación de este hecho, obtenemos una versión del teorema de multiplicadores de Meyer y a través de este teorema y derivadas fraccionales, obtenemos una caracterización de espacios Dunkl-potenciales.

Keywords and Phrases: Dunkl-Ornstein-Uhlenbeck operator, generalized Hermite polynomial, squared field operator, Meyer's multiplier theorem, Dunkl-potential space, fractional integral, fractional derivative.

2010 AMS Mathematics Subject Classification: 33C45, 6A33, 33C52.

${ }^{1}$ The author's research was partially supported by DID-USB-CB-004-17 


\section{Preliminaries}

In this section we collect some notations and results in the Dunkl theory (see [5]), but particularly for the $\mathbb{Z}_{2}^{\mathrm{d}}$ group.

Let $v=\left(v_{1}, \ldots, v_{d}\right) \in \mathbb{Z}_{+}^{d}$ be a multi-index, where $\mathbb{Z}_{+}=\{0,1,2, \ldots\}$, so $v !=\prod_{j=1}^{d} v_{j}$ ! and $|v|=\sum_{j=1}^{d} v_{j}$. For $x=\left(x_{1}, \ldots, x_{d}\right) \in \mathbb{R}^{d}$, we set $x^{v}=x_{1}^{v_{1}} \ldots x_{d}^{v_{d}}$ and $|x|_{2}^{2}=\sum_{j=1}^{d} x_{j}^{2}$. In what follows, we denote $\partial_{j}=\partial / \partial x_{j}$, for each $1 \leq j \leq d$, and $\partial^{v}=\partial_{1}^{v_{1}} \ldots \partial_{d}^{v_{d}}$. Also, $\langle.,$.$\rangle denotes the$ Euclidean inner product in $\mathbb{R}^{\mathrm{d}}$ and finally, $\triangle$ and $\nabla$ denote the usual Laplacean and the usual gradient, respectively.

Let us consider the finite reflection group generated by

$$
\sigma_{j}(x)=x-2 \frac{\left\langle x, e_{j}\right\rangle}{\left|e_{j}\right|_{2}^{2}} e_{j}
$$

where $\left(e_{j}\right)_{j=1}^{d}$ are the standard unit vectors of $\mathbb{R}^{d}$. So, for each $j=1, \ldots, d$,

$$
\sigma_{j}\left(x_{1}, \ldots, x_{j}, \ldots, x_{d}\right)=\left(x_{1}, \ldots,-x_{j}, \ldots, x_{d}\right)
$$

and isomorphic to $\mathbb{Z}_{2}^{\mathrm{d}}=\{0,1\}^{\mathrm{d}}$. The reflection $\sigma_{j}$ is in the hyperplane orthogonal to $e_{j}$. Then, we consider the root system $\mathrm{R}$ and the positive root system $\mathrm{R}_{+}$, respectively, as

$$
R=\left\{ \pm \sqrt{2} e_{j}: j=1, \ldots, d\right\}, \quad R_{+}=\left\{\sqrt{2} e_{j}: j=1, \ldots, d\right\}
$$

and let $k$ be a nonnegative multiplicity function $k: R_{+} \rightarrow[0, \infty)$, which is $\mathbb{Z}_{2}^{d}$-invariant. Then, we set $k=\left(k_{1}, \ldots, k_{d}\right)$, where $k_{j}=\alpha_{j}+(1 / 2)$ and $\alpha_{j} \geq-1 / 2$, for each $j=1, \ldots, d$.

Thus, in this particular case, the Dunkl differential difference operators, $\mathrm{T}_{j}^{\mathrm{k}}$, are given by

$$
T_{j}^{k} f(x)=\partial_{j} f(x)+k_{j}\left(\frac{f(x)-f\left(\sigma_{j} x\right)}{x_{j}}\right), \quad j=1, \ldots, d
$$

with $f \in C^{1}\left(\mathbb{R}^{d}\right)$ and in the following, the operator

$$
\triangle_{k}=\sum_{j=1}^{d}\left(T_{j}^{k}\right)^{2}
$$

given explicitty by

$$
\triangle_{k} f(x)=\sum_{j=1}^{d}\left\{\partial_{j}^{2} f(x)+\frac{2 k_{j}}{x_{j}} \partial_{j} f(x)-k_{j}\left(\frac{f(x)-f\left(\sigma_{j} x\right)}{x_{j}^{2}}\right)\right\}
$$

is called "the generalized Laplacian" or "Dunkl-Laplacian" associated to $\mathbb{Z}_{2}^{\mathrm{d}}$ and k.

Then the Dunkl-Ornstein-Uhlenbeck differential operator is defined as,

$$
\mathrm{L}_{\mathrm{k}}=\frac{\triangle_{\mathrm{k}}}{2}-\left\langle x, \nabla_{\mathrm{x}}\right\rangle
$$


and therefore, from (1.1), the Dunkl-Ornstein-Uhlenbeck differential operator can be written as

$$
L_{k} f(x)=\sum_{j=1}^{d} \frac{1}{2}\left\{\partial_{j}^{2} f(x)+\frac{2 k_{j}}{x_{j}} \partial_{j} f(x)-k_{j}\left(\frac{f(x)-f\left(\sigma_{j} x\right)}{x_{j}^{2}}\right)\right\}-x_{j} \partial_{j} f(x) .
$$

Here, the corresponding weight function is defined by $w_{k}(x)=\prod_{j=1}^{d}\left|x_{j}\right|^{2 k_{j}}$ and we consider the Hilbert space $L^{2}\left(m_{k}\right)$, where the probability measure, $m_{k}$, is defined by $m_{k}(d x)=$ $c_{k} \exp \left(-|x|_{2}^{2}\right) w_{k}(x) d x$, with $x \in \mathbb{R}^{d}$ and $c_{k}=\left(\int_{\mathbb{R}^{d}} \exp \left(-|x|_{2}^{2}\right) w_{k}(x) d x\right)^{-1}$.

Now, we consider a complete system of orthogonal polynomials, with respect to the measure $m_{k}$, which is known as generalized Hermite polynomials. In dimension one, for the reflection group $\mathbb{Z}_{2}$, the corresponding generalized Hermite polynomials are defined as

$$
\left\{\begin{array}{l}
H_{2 n}^{k}(x)=(-1)^{n} 2^{2 n} n ! L_{n}^{\alpha-(1 / 2)}\left(x^{2}\right) \\
H_{2 n+1}^{k}(x)=(-1)^{n} 2^{2 n+1} n ! x L_{n}^{\alpha+(1 / 2)}\left(x^{2}\right)
\end{array}\right.
$$

where $L_{n}^{\alpha}$ are the Laguerre polynomials of degree $n$ and order $\alpha$, (see [11 and [4, pages 156,157]). In the multidimensional case the generalized Hermite polynomials are defined by taking tensor products of the one-dimensional $H_{n}^{k}$; that is, $H_{v}^{k}(x)=\prod_{j=1}^{d} H_{v_{j}}^{k_{j}}\left(x_{j}\right), x \in \mathbb{R}^{d}, v \in \mathbb{Z}_{+}^{d}$. This way, we will denote

$$
h_{v}^{k}=2^{-|v| / 2} H_{v}^{k}, \quad v \in \mathbb{Z}_{+}^{\mathrm{d}},
$$

from now on.

$H_{v}^{k}$ is a polynomial of degree $|v|$ and $\left\{h_{v}^{k}\right\}_{v \in \mathbb{Z}_{+}^{d}}$ forms an orthonormal basis of $L^{2}\left(m_{k}\right)$. The generalized Hermite polynomials satisfy the following important identity which is known as Mehler's formula. For $r \in \mathbb{C}$ with $|r|<1$,

$$
\sum_{v \in \mathbb{Z}_{+}^{\mathrm{d}}} h_{v}^{\mathrm{k}}(x) h_{v}^{\mathrm{k}}(y) r^{|v|}=\frac{1}{\left(1-r^{2}\right)^{|k|+d / 2}} \exp \left(-\frac{r^{2}\left(|x|_{2}^{2}+|y|_{2}^{2}\right)}{1-r^{2}}\right) E_{k}\left(\frac{2 r x}{1-r^{2}}, y\right)
$$

where the sum is absolutely convergent and the Dunkl kernel, $E_{k}(x, y)$, replaces the usual exponential function, $\exp \langle x, y\rangle$. Then, from [11] the generalized Hermite polynomials are eigenfunctions of $\mathrm{L}_{\mathrm{k}}$

$$
\mathrm{L}_{\mathrm{k}}\left(\mathrm{h}_{v}^{\mathrm{k}}\right)=-|v| \mathrm{h}_{v}^{\mathrm{k}}, \quad \forall v \in \mathbb{Z}_{+}^{\mathrm{d}} .
$$

Also, let $C_{n}^{k}$ be the closed subspace of $L^{2}\left(m_{k}\right)$ generate by linear combination of $\left\{h_{v}^{k}:|v|=n\right\}$ and we denote by $J_{\mathfrak{n}}^{k}$ the orthogonal projection of $L^{2}\left(m_{k}\right)$ onto $C_{n}^{k}$. If $f$ is a polynomial, then

$$
J_{n}^{k} f=\sum_{|v|=n} c_{v}^{k}(f) h_{v}^{k}
$$

where given a function $f \in L^{2}\left(m_{k}\right)$, its Dunkl-Fourier coefficient is defined by $c_{v}^{k}(f)=\int_{\mathbb{R}^{d}} f(x) h_{v}^{k}(x) m_{k}(d x)$ and therefore, if $f \in L^{2}\left(m_{k}\right)$, its Dunkl-Hermite expansion is given by $f=\sum_{n=0}^{\infty} J_{n}^{k} f$. Thus, the operator

$$
\mathrm{L}_{k} f=\sum_{n=0}^{\infty}-n J_{n}^{k} f
$$


defined on the domain $D_{2}\left(L_{k}\right)=\left\{f \in L^{2}\left(m_{k}\right): \sum_{n=0}^{\infty} \sum_{|v|=n}\left|c_{v}^{k}(f)\right|^{2}<\infty\right\}$, is a self-adjoint extension of $L_{k}$ considered on $C_{c}^{\infty}\left(\mathbb{R}^{d}\right)$. More precisely, $L_{k}$ has a clousure which also will be denoted by $L_{k}$.

Now, following [11, 13, the generalized heat kernel, $\Gamma_{k}(t, x, y)$, is given by

$$
\Gamma_{k}(t, x, y)=\frac{c_{k} \exp \left\{-\left(|x|_{2}^{2}+|y|_{2}^{2}\right) / 4 t\right\}}{(4 t)^{|k|+d / 2}} E_{k}\left(\frac{x}{\sqrt{2 t}}, \frac{y}{\sqrt{2 t}}\right)
$$

where $x, y \in \mathbb{R}^{\mathrm{d}}$ and $t>0$. Therefore, from [12] the Dunkl-Ornstein-Uhlenbeck integral operator is defined as

$$
\mathrm{O}_{\mathrm{t}}^{\mathrm{k}} \mathrm{f}(\mathrm{x})=\int_{\mathbb{R}^{\mathrm{d}}} \Gamma_{\mathrm{k}}\left(\frac{\left(1-e^{-2 \mathrm{t}}\right)}{4}, e^{-\mathrm{t}} x, y\right) f(y) w_{k}(y) d y .
$$

But, (1.4) allows us to express

$$
\exp \left(|y|_{2}^{2}\right) \Gamma_{k}\left(\frac{\left(1-e^{-2 t}\right)}{4}, e^{-t} x, y\right)=\frac{c_{k} \exp \left(-\frac{e^{-2 t}\left(|x|_{2}^{2}+|y|_{2}^{2}\right)}{1-e^{-2 t}}\right)}{\left(1-e^{-2 t}\right)^{|k|+d / 2}} E_{k}\left(\frac{\sqrt{2} e^{-t} x}{\sqrt{1-e^{-2 t}}}, \frac{\sqrt{2} e^{-t} y}{\sqrt{1-e^{-2 t}}}\right),
$$

and since, $E_{k}\left(\frac{\sqrt{2} e^{-t} x}{\sqrt{1-e^{-2 t}}}, \frac{\sqrt{2} e^{-t} y}{\sqrt{1-e^{-2 t}}}\right)=E_{k}\left(\frac{2 e^{-t} x}{1-e^{-2 t}}, y\right)$, by using Mehler formula, we get explicity:

$$
\mathrm{O}_{\mathrm{t}}^{\mathrm{k}} \mathrm{f}(\mathrm{x})=\int_{\mathbb{R}^{\mathrm{d}}} \mathrm{f}(\mathrm{y}) \mathrm{O}_{\mathrm{t}}^{\mathrm{k}}(x, y) \mathrm{m}_{\mathrm{k}}(\mathrm{d} y)
$$

where, $\mathrm{O}_{\mathrm{t}}^{k}(x, y)=\sum_{v \in \mathbb{Z}_{+}^{\mathrm{d}}} e^{-|v| t} h_{v}^{k}(x) h_{v}^{k}(y)$. Besides, $\left\{\mathrm{O}_{\mathrm{t}}^{k}\right\}_{t \geq 0}$ is a positive, strongly continuous contraction semigroup on $C_{0}\left(\mathbb{R}^{d}\right)$ with generator $L_{k}$. Thus, formally, we write $O_{t}^{k}=e^{\left(t L_{k}\right)}$ and following [12, 14, if we consider

$$
M_{t}^{k}(x, d y)=\Gamma_{k}\left(\frac{\left(1-e^{-2 t}\right)}{4}, e^{-t} x, y\right) w_{k}(y) d y,
$$

form, (together with the trivial kernel $M_{0}^{k}$ ), a semigroup of Markov kernels.

Also, the corresponding Dunkl-Poisson semigroup $\left\{\mathrm{P}_{\mathrm{t}}^{k}\right\}_{\mathrm{t} \geq 0}$ is defined, by means of subordination principle, as

$$
P_{t}^{k} f(x)=\frac{1}{\sqrt{\pi}} \int_{0}^{\infty} \frac{\exp (-u)}{\sqrt{u}} O_{t^{2} / 4 u}^{k} f(x) d u=\int_{\mathbb{R}^{d}} f(y) P_{t}^{k}(x, y) m_{k}(d y),
$$

where the kernel $P_{t}^{k}(x, y)$ is defined as

$$
P_{t}^{k}(x, y)=\frac{1}{\sqrt{\pi}} \int_{0}^{\infty} \frac{\exp (-u)}{\sqrt{u}} O_{t^{2} / 4 u}^{k}(x, y) d u
$$

Again, $\left\{P_{t}^{k}\right\}_{t \geq 0}$ is a positive, strongly continuous semigroup with infinitesimal generator $\left(-L_{k}\right)^{1 / 2}$ and it is a Markov process (see [12, 16]). In particular, by (1.3) we obtain that

$$
\mathrm{O}_{\mathrm{t}}^{\mathrm{k}}\left(\mathrm{h}_{v}^{\mathrm{k}}\right)=e^{-|v| t} h_{v}^{k} \text { and } \mathrm{P}_{\mathrm{t}}^{\mathrm{k}}\left(\mathrm{h}_{v}^{\mathrm{k}}\right)=e^{-\mathrm{t} \sqrt{|v|}} h_{v}^{\mathrm{k}}
$$


also, if $f$ is a polynomial

$$
\mathrm{O}_{\mathrm{t}}^{k} f=\sum_{n \geq 0} e^{-n t} J_{n}^{k} f \quad \text { and } \quad P_{t}^{k} f=\sum_{n \geq 0} e^{-t \sqrt{n}} J_{n}^{k} f
$$

Following [2, 3], let us consider the squared field operator, $\Gamma$, associated to $\mathrm{L}_{k}$, as

$$
\Gamma(f, g)=\frac{1}{2}\left[L_{k}(f g)-f L_{k}(g)-g L_{k}(f)\right], \quad \forall f, g \in \mathcal{A},
$$

where we choose $\mathcal{A}$ as the set of all polynomials on $\mathbb{R}^{\mathrm{d}}$, which is a dense subspace in $\mathrm{L}^{2}\left(\mathrm{~m}_{\mathrm{k}}\right)$. Besides, let us consider the operator $\Gamma_{2}$ defined as

$$
\Gamma_{2}(f, g)=\frac{1}{2}\left[L_{k} \Gamma(f, g)-\Gamma\left(f, L_{k} g\right)-\Gamma\left(g, L_{k} f\right)\right], \quad \forall f, g \in \mathcal{A} \times \mathcal{A}
$$

and throughout this paper we denote $\Gamma(f)=\Gamma(f, f)$ and $\Gamma_{2}(f)=\Gamma_{2}(f, f)$.

Again, motivated by [2, 3, we say that the differential operator $L_{k}$ satisfies a $C D(\rho, n)$ inequality, (a curvature-dimension inequality with curvature $\rho$ and dimension $n$ ), if and only if

$$
\Gamma_{2}(f) \geq \rho \Gamma(f)+\frac{1}{n}\left(L_{k} f\right)^{2}, \quad \forall f \in \mathcal{A},
$$

where $\rho \in \mathbb{R}$ and $n \in[1, \infty]$. Particularly, $L_{k}$ satisfies a $C D(\rho, \infty)$-inequality, if and only if

$$
\Gamma_{2}(\mathbf{f}) \geq \rho \Gamma(f) \quad \forall f \in \mathcal{A} \text {. }
$$

Finally, we denote a Dirichley form associated to the measure $m_{k}$ by

$$
\mathcal{E}(f)=\int \Gamma(f)(x) m_{k}(d x)
$$

and the Entropy of a positive function $f$ as

$$
\operatorname{Ent}(f)=\int f(x) \log (f)(x) m_{k}(d x)-\int f(x) m_{k}(d x) \log \left(\int f(x) m_{k}(d x)\right) .
$$

In this case, a logarithmic Sobolev inequality, $\operatorname{LS}(A, C)$, has the form

$$
\operatorname{Ent}\left(f^{2}\right) \leq A \int f^{2}(x) m_{k}(d x)+C \mathcal{E}(f), \forall f \in \mathcal{A} .
$$

Particularly, if $A=0$ we say that the logarithmic Sobolev inequality is tight. The logarithmic Sobolev inequalities relate Entropy to the Dirichlet norm (the Energy) and these type of inequalities were introduced by L. Gross to study the hypercontractive propertie of the diffusion semigroups and the Markov semigroups, (see [7, 8]).

\section{The results}

Now, we are ready to present the results of this paper. 


\subsection{Hypercontractivity of Dunkl-Ornstein-Uhlenbeck semigroup}

Following D. Bakry [2, 3], we turn now to the study of the local structure of the Dunkl-OrnsteinUhlenbeck differential operator $L_{k}$. Then, we started recalling the operators, $\Gamma$ and $\Gamma_{2}$, defined in (1.6) and (1.7) respectively. That is,

$$
\Gamma(f)=\frac{1}{2}\left[L_{k}\left(f^{2}\right)-2 f L_{k}(f)\right] \quad \text { and } \quad \Gamma_{2}(f)=\frac{1}{2}\left[L_{k} \Gamma(f)-2 \Gamma\left(f, L_{k} f\right)\right],
$$

where,

$$
\Gamma\left(f, L_{k} f\right)=\frac{1}{2}\left[L_{k}\left(f L_{k} f\right)-f L_{k}\left(L_{k} f\right)-\left(L_{k} f\right)^{2}\right],
$$

$\forall \mathrm{f} \in \mathcal{A}$. Again, we consider $\mathcal{A}$ as the space of all polynomials on $\mathbb{R}^{\mathrm{d}}$.

Now, from (1.2), let us denote

$$
\mathrm{L}_{\mathrm{k}} \mathrm{f}=\sum_{j=1}^{\mathrm{d}} \mathrm{L}_{\mathrm{k}}^{\mathrm{j}} \mathrm{f}, \quad \forall \mathrm{f} \in \mathcal{A}
$$

where, for each $j=1, \ldots, d$,

$$
L_{k}^{j} f(x)=\frac{1}{2}\left\{\partial_{j}^{2} f(x)+\frac{2 k_{j}}{x_{j}} \partial_{j} f(x)-k_{j}\left(\frac{f(x)-f\left(\sigma_{j} x\right)}{x_{j}^{2}}\right)\right\}-x_{j} \partial_{j} f(x) .
$$

Thus, in order to obtain our results, we prove the following technical Lemmas.

Lemma 2.1. Let $\mathrm{L}_{\mathrm{k}}$ be the Dunkl-Ornstein-Uhlenbeck differential operator defined as in (1.2). Then

$$
\Gamma(f)(x)=\frac{|\nabla f(x)|^{2}}{2}+\sum_{j=1}^{d} k_{j}\left(\frac{f(x)-f\left(\sigma_{j} x\right)}{2 x_{j}}\right)^{2}, \quad \forall f \in \mathcal{A} .
$$

Proof. From (2.1) and (2.3), it is obvious that we can write

$$
\Gamma(f)(x)=\frac{1}{2} \sum_{j=1}^{d}\left\{L_{k}^{j}\left(f^{2}\right)(x)-2 f(x) L_{k}^{j}(f)(x)\right\},
$$

where, considering (2.4), we denote

$$
L_{k}^{j} f(x)=L^{j} f(x)+\Omega_{k}^{j} f(x), \quad j=1, \ldots, d
$$

with

$$
L^{j} f(x)=\frac{1}{2} \partial_{j}^{2} f(x)-x_{j} \partial f(x) \quad \text { and } \quad \Omega_{k}^{j} f(x)=\frac{1}{2}\left[\frac{2 k_{j}}{x_{j}} \partial_{j} f(x)-\frac{k_{j}}{x_{j}^{2}}\left(f(x)-f\left(\sigma_{j} x\right)\right)\right] \text {, }
$$


for each $j=1, \ldots, d$. Thus, first we have to compute $L^{j}\left(f^{2}\right)$ and $2 f L^{j}(f)$. We can see that

$$
\left\{\begin{array}{l}
L^{j}\left(f^{2}\right)(x)=\left(\partial_{j} f\right)^{2}(x)+f(x) \partial_{j}^{2} f(x)-2 x_{j} f(x) \partial_{j} f(x), \\
2 f(x) L^{j}(f)(x)=f(x) \partial_{j}^{2} f(x)-2 x_{j} f(x) \partial_{j} f(x)
\end{array}\right.
$$

since

$$
\left\{\begin{array}{l}
\partial_{j}\left(f^{2}\right)(x)=2 f(x) \partial_{j} f(x) \\
\partial_{j}^{2}\left(f^{2}\right)(x)=2\left(\partial_{j} f\right)^{2}(x)+2 f(x) \partial_{j}^{2} f(x)
\end{array}\right.
$$

and therefore

$$
L^{j}\left(f^{2}\right)(x)-2 f(x) L^{j} f(x)=\left(\partial_{j} f\right)^{2}(x) .
$$

On the other hand,

$$
\Omega_{k}^{j}\left(f^{2}\right)(x)=\frac{1}{2}\left[\frac{4 k_{j}}{x_{j}} f(x) \partial_{j} f(x)-\frac{k_{j}}{x_{j}^{2}}\left(f^{2}(x)-f^{2}\left(\sigma_{j} x\right)\right)\right]
$$

and

$$
2 f(x) \Omega_{k}^{j} f(x)=\frac{1}{2}\left[\frac{4 k_{j}}{x_{j}} f(x) \partial_{j} f(x)-\frac{k_{j}}{x_{j}^{2}}\left(2 f^{2}(x)-2 f(x) f\left(\sigma_{j} x\right)\right)\right] .
$$

Then, we obtain that

$$
\Omega_{k}^{j}\left(f^{2}\right)(x)-2 f(x) \Omega_{k}^{j}\left(f^{2}\right)(x)=\frac{k_{j}}{2 x_{j}^{2}}\left(f(x)-f\left(\sigma_{j} x\right)\right)^{2}
$$

and consequently, the result of the Lemma follows from (2.5) and (2.6), since

$$
\begin{aligned}
\Gamma(f)(x) & =\frac{1}{2} \sum_{j=1}^{d}\left\{L^{j}\left(f^{2}\right)(x)-2 f(x) L^{j} f(x)\right\}+\left\{\Omega_{k}^{j}\left(f^{2}\right)(x)-2 f(x) \Omega_{k}^{j} f(x)\right\} \\
& =\frac{1}{2}\left\{\sum_{j=1}^{d}\left(\partial_{j} f\right)^{2}(x)+\frac{k_{j}}{2 x_{j}^{2}}\left(f(x)-f\left(\sigma_{j} x\right)\right)^{2}\right\} .
\end{aligned}
$$

Lemma 2.2. Let $\mathrm{L}_{\mathrm{k}}$ be the Dunkl-Ornstein-Uhlenbeck differential operator defined as in (1.2). Then, $\forall \mathrm{f} \in \mathcal{A}$, we have

$$
\begin{aligned}
L_{k}\left(f L_{k} f\right)(x)=\left(L_{k} f\right)^{2}(x)+f(x) L_{k}\left(L_{k} f\right)(x) & +\left\langle\nabla f(x), \nabla L_{k} f(x)\right\rangle \\
& +\frac{1}{2} \sum_{i=1}^{d} \sum_{j=1}^{d} \frac{k_{i}}{x_{i}^{2}}\left(f(x)-f\left(\sigma_{i} x\right)\right)\left(L_{k}^{j} f(x)-L_{k}^{j} f\left(\sigma_{i} x\right)\right) .
\end{aligned}
$$

Proof. From (2.3) we obtain that

$$
L_{k}\left(f L_{k} f\right)(x)=\sum_{i=1}^{d} \sum_{j=1}^{d} L_{k}^{i}\left(f L_{k}^{j} f\right)(x)
$$


and by using (2.4) with $\mathrm{fL}_{k}^{j} f$ instead of $f$, we can express

$$
\begin{aligned}
& L_{k}^{i}\left(f L_{k}^{j} f\right)(x)= \\
& \frac{1}{2}\left[\partial_{i}^{2}\left(f L_{k}^{j} f\right)(x)+\frac{2 k_{i}}{x_{i}} \partial_{i}\left(f L_{k}^{j} f\right)(x)-\frac{k_{i}}{x_{i}^{2}}\left(f(x) L_{k}^{j} f(x)-f\left(\sigma_{i} x\right) L_{k}^{j} f\left(\sigma_{i} x\right)\right)\right] \\
& -x_{i} \partial_{i}\left(f L_{k}^{j} f\right)(x) .
\end{aligned}
$$

Now, for each $j=1, \ldots, d$ and $i=1, \ldots, d$, we have

$$
\left\{\begin{array}{l}
\partial_{i}\left(f L_{k}^{j} f\right)(x)=\partial_{i} f(x) L_{k}^{j} f(x)+f(x) \partial_{i}\left(L_{k}^{j} f\right)(x), \\
\partial_{i}^{2}\left(f L_{k}^{j} f\right)(x)=\partial_{i}^{2} f(x) L_{k}^{j} f(x)+2 \partial_{i} f(x) \partial_{i}\left(L_{k}^{j} f\right)(x)+f(x) \partial_{i}^{2}\left(L_{k}^{j} f\right)(x)
\end{array}\right.
$$

and since,

$$
\begin{aligned}
-\frac{k_{i}}{x_{i}^{2}}\left[f(x) L_{k}^{j} f(x)\right. & \left.-f\left(\sigma_{i} x\right) L_{k}^{j} f\left(\sigma_{i} x\right)\right]= \\
& -\frac{k_{i}}{x_{i}^{2}}\left(f(x)-f\left(\sigma_{i} x\right)\right) L_{k}^{j} f(x)-\frac{k_{i}}{x_{i}^{2}} f(x)\left(L_{k}^{j} f(x)-L_{k}^{j} f\left(\sigma_{i} x\right)\right) \\
& +\frac{k_{i}}{x_{i}^{2}}\left(f(x)-f\left(\sigma_{i} x\right)\right)\left(L_{k}^{j} f(x)-L_{k}^{j} f\left(\sigma_{i} x\right)\right),
\end{aligned}
$$

then substituing (2.8) and (2.9) in (2.7) we obtain explicitly

$$
\begin{aligned}
& L_{k}^{i}\left(f L_{k}^{j} f\right)(x)= \\
& \left(\frac{1}{2}\left[\partial_{i}^{2} f(x)+\frac{2 k_{i}}{x_{i}} \partial_{i} f(x)-\frac{k_{i}}{x_{i}^{2}}\left(f(x)-f\left(\sigma_{i} x\right)\right)\right]-x_{i} \partial_{i} f(x)\right) L_{k}^{j} f(x)+ \\
& \left(\frac{1}{2}\left[\partial_{i}^{2}\left(L_{k}^{j} f\right)(x)+\frac{2 k_{i}}{x_{i}} \partial_{i}\left(L_{k}^{j} f\right)(x)-\frac{k_{i}}{x_{i}^{2}}\left(L_{k}^{j} f(x)-L_{k}^{j} f\left(\sigma_{i} x\right)\right)\right]-x_{i} \partial_{i}\left(L_{k}^{j} f\right)(x)\right) f(x)+ \\
& \partial_{i} f(x) \partial_{i}\left(L_{k}^{j} f\right)(x)+\frac{k_{i}}{2 x_{i}^{2}}\left(f(x)-f\left(\sigma_{i} x\right)\right)\left(L_{k}^{j} f(x)-L_{k}^{j} f\left(\sigma_{i} x\right)\right)
\end{aligned}
$$

and we can express

$$
\begin{aligned}
& \mathrm{L}_{\mathrm{k}}^{i}\left(f \mathrm{~L}_{\mathrm{k}}^{\mathrm{j}} f\right)(x)= \\
& L_{k}^{i} f(x) L_{k}^{j} f(x)+f(x) L_{k}^{i}\left(L_{k}^{j} f\right)(x)+\partial_{i} f(x) \partial_{i}\left(L_{k}^{j} f\right)(x)+ \\
& \frac{k_{i}}{2 x_{i}^{2}}\left(f(x)-f\left(\sigma_{i} x\right)\right)\left(L_{k}^{j} f(x)-L_{k}^{j} f\left(\sigma_{i} x\right)\right),
\end{aligned}
$$

for each $j=1, \ldots, d$ and $i=1, \ldots, d$. Therefore, taking the sum with respect to $i$ and $j$, we obtain the result of the Lemma. 
Consequently, the identity (2.2) and the Lemma 2.2 allows us to write

$$
\begin{aligned}
\Gamma_{2}(f)(x)=\frac{1}{2}\left[L_{k}(\Gamma(f))(x)-\langle\nabla f(x),\right. & \left.\nabla L_{k} f(x)\right\rangle \\
& \left.-\sum_{i=1}^{d} \sum_{j=1}^{d} \frac{k_{i}}{2 x_{i}^{2}}\left(f(x)-f\left(\sigma_{i} x\right)\right)\left(L_{k}^{j} f(x)-L_{k}^{j} f\left(\sigma_{i} x\right)\right)\right]
\end{aligned}
$$

and in the following result we obtain an explicit expression for the operator $\Gamma_{2}(f)$. More precisely,

Proposition 2.3. Let $\mathrm{L}_{\mathrm{k}}$ be the Dunkl-Ornstein-Uhlenbeck differential operator defined as in (1.2). Then, $\forall \mathbf{f} \in \mathcal{A}$, the operator $\Gamma_{2}(\mathbf{f})$ can be rewritten as

$$
\begin{aligned}
\Gamma_{2}(f)(x)= & \sum_{i=1}^{d}\left\{\frac{\left(\partial_{i}^{2} f\right)^{2}(x)}{4}+\frac{\left(\partial_{i} f\right)^{2}(x)}{2}+\frac{k_{i}}{4}\left[\frac{\left(\partial_{i} f(x)+\partial_{i} f\left(\sigma_{i} x\right)\right)}{x_{i}}-\frac{\left(f(x)-f\left(\sigma_{i} x\right)\right)}{x_{i}^{2}}\right]^{2}\right. \\
& \left.+\frac{k_{i}}{2}\left[\frac{\partial_{i} f(x)}{x_{i}}-\frac{\left(f(x)-f\left(\sigma_{i} x\right)\right)}{2 x_{i}^{2}}\right]^{2}+\frac{k_{i}}{4 x_{i}^{2}}\left(f(x)-f\left(\sigma_{i} x\right)\right)^{2}\right\} \\
& +\sum_{i=1}^{d} \sum_{j=1, j \neq i}^{d}\left\{\frac{\left(\partial_{i j}^{2} f\right)^{2}(x)}{4}+\frac{k_{i}}{8 x_{i}^{2}}\left(\partial_{j} f(x)-\partial_{j} f\left(\sigma_{i} x\right)\right)^{2}+\frac{k_{j}}{8 x_{j}^{2}}\left(\partial_{i} f(x)-\partial_{i} f\left(\sigma_{j} x\right)\right)^{2}\right. \\
& \left.+\frac{k_{i} k_{j}}{16 x_{i}^{2} x_{j}^{2}}\left[\left(f(x)-f\left(\sigma_{i} x\right)\right)-\left(f\left(\sigma_{j} x\right)-f\left(\sigma_{i} \sigma_{j} x\right)\right)\right]^{2}\right\} .
\end{aligned}
$$

Proof. We have to compute each term in equation (2.10). As first step in this argument, observe that by using (2.3) and the Lemma 2.1 we get

$$
L_{k}(\Gamma f)=\sum_{i=1}^{d} \sum_{j=1}^{d} L_{k}^{j}\left(\Gamma_{i} f\right)=\sum_{i=1}^{d} L_{k}^{i}\left(\Gamma_{i} f\right)+\sum_{i=1}^{d} \sum_{j=1, j \neq i}^{d} L_{k}^{j}\left(\Gamma_{i} f\right),
$$

where we denote

$$
\Gamma_{i} f(x)=\frac{\left(\partial_{i} f\right)^{2}(x)}{2}+k_{i}\left(\frac{f(x)-f\left(\sigma_{i} x\right)}{2 x_{i}}\right)^{2} .
$$

Using the identity (2.4) with $\Gamma_{i} f$ instead of $f$, we can express

$$
L_{k}^{j}\left(\Gamma_{i} f\right)(x)=\frac{1}{2}\left\{\partial_{j}^{2}\left(\Gamma_{i} f\right)(x)+\frac{2 k_{j}}{x_{j}} \partial_{j}\left(\Gamma_{i} f\right)(x)-k_{j}\left(\frac{\Gamma_{i} f(x)-\Gamma_{i} f\left(\sigma_{j} x\right)}{x_{j}^{2}}\right)\right\}-x_{j} \partial_{j} \Gamma_{i} f(x) .
$$

Then, let us consider two cases: $\mathfrak{i}=\mathfrak{j}$ and $i \neq j$, with $1 \leq \mathfrak{i} \leq \mathrm{d}$ and $1 \leq \mathfrak{j} \leq \mathrm{d}$. If $\mathfrak{i}=\mathfrak{j}$, differentiating (2.11) with respect to $x_{i}$ we obtain

$$
\partial_{i}\left(\Gamma_{i} f\right)(x)=\partial_{i} f(x) \partial_{i}^{2} f(x)+\frac{k_{i}}{2 x_{i}^{2}}\left(f(x)-f\left(\sigma_{i} x\right)\right)\left(\partial_{i} f(x)+\partial_{i} f\left(\sigma_{i} x\right)\right)-\frac{k_{i}}{2 x_{i}^{3}}\left(f(x)-f\left(\sigma_{i} x\right)\right)^{2}
$$


and

$$
\begin{aligned}
& \partial_{i}^{2}\left(\Gamma_{i} f\right)(x)=\partial_{i} f(x) \partial_{i}^{3} f(x)+\left(\partial_{i}^{2} f\right)^{2}(x)+\frac{k_{i}}{2 x_{i}^{2}}\left(\partial_{i} f(x)+\partial_{i} f\left(\sigma_{i} x\right)\right)^{2} \\
& +\frac{k_{i}}{2 x_{i}^{2}}\left(f(x)-f\left(\sigma_{i} x\right)\right)\left(\partial_{i}^{2} f(x)-\partial_{i}^{2} f\left(\sigma_{i} x\right)\right)-\frac{2 k_{i}}{x_{i}^{3}}\left(f(x)-f\left(\sigma_{i} x\right)\right)\left(\partial_{i} f(x)+\partial_{i} f\left(\sigma_{i} x\right)\right) \\
& +\frac{3 k_{i}}{2 x_{i}^{4}}\left(f(x)-f\left(\sigma_{i} x\right)\right)^{2}
\end{aligned}
$$

(note that $\left.\partial_{i}\left(\sigma_{i} x\right)=-1\right)$.

Otherwise, considering (2.11) with $\sigma_{i} x$ instead of $x$, we can write

$$
\Gamma_{i} f\left(\sigma_{i} x\right)=\frac{\left(\partial_{i} f\right)^{2}\left(\sigma_{i} x\right)}{2}+k_{i}\left(\frac{f\left(\sigma_{i} x\right)-f(x)}{2 x_{i}}\right)^{2}, \quad 1 \leq i \leq d
$$

since $\sigma_{i}\left(\sigma_{i} x\right)=x$ and we obtain that

$$
\frac{k_{i}}{2 x_{i}^{2}}\left(\Gamma_{i} f(x)-\Gamma_{i} f\left(\sigma_{i} x\right)\right)=\frac{k_{i}}{4 x_{i}^{2}}\left(\left(\partial_{i} f\right)^{2}(x)-\left(\partial_{i} f\right)^{2}\left(\sigma_{i} x\right)\right) .
$$

Therefore, if $i=j$, replacing (2.13), (2.14) and (2.15) in (2.12), we get that

$$
\begin{aligned}
& L_{k}^{i}\left(\Gamma_{i} f\right)(x)= \\
& \frac{\left(\partial_{i}^{2} f\right)^{2}(x)}{2}+\frac{\partial_{i} f(x) \partial_{i}^{3} f(x)}{2}-x_{i} \partial_{i}^{2} f(x) \partial_{i} f(x)+\frac{k_{i}}{4 x_{i}^{2}}\left(\partial_{i} f(x)+\partial_{i} f\left(\sigma_{i} x\right)\right)^{2} \\
& +\frac{k_{i}}{4 x_{i}^{2}}\left(f(x)-f\left(\sigma_{i} x\right)\right)\left(\partial_{i}^{2} f(x)-\partial_{i}^{2} f\left(\sigma_{i} x\right)\right)-\frac{k_{i}}{4 x_{i}^{2}}\left(\left(\partial_{i} f\right)^{2}(x)-\left(\partial_{i} f\right)^{2}\left(\sigma_{i} x\right)\right) \\
& +\left[\frac{k_{i}^{2}}{2 x_{i}^{3}}-\frac{k_{i}}{x_{i}^{3}}-\frac{k_{i}}{2 x_{i}}\right]\left(f(x)-f\left(\sigma_{i} x\right)\right)\left(\partial_{i} f(x)+\partial_{i} f\left(\sigma_{i} x\right)\right)+\frac{k_{i}}{x_{i}} \partial_{i}^{2} f(x) \partial_{i} f(x) \\
& +\left[\frac{3 k_{i}}{4 x_{i}^{4}}-\frac{k_{i}^{2}}{2 x_{i}^{4}}+\frac{k_{i}}{2 x_{i}^{2}}\right]\left(f(x)-f\left(\sigma_{i} x\right)\right)^{2} .
\end{aligned}
$$

Now, if $i \neq j$, again differentiating (2.11) with respect to $x_{j}$ we obtain

$$
\partial_{j}\left(\Gamma_{i} f\right)(x)=\partial_{i} f(x) \partial_{i j}^{2} f(x)+\frac{k_{i}}{2 x_{i}^{2}}\left(f(x)-f\left(\sigma_{i} x\right)\right)\left(\partial_{j} f(x)-\partial_{j} f\left(\sigma_{i} x\right)\right)
$$

and

$$
\begin{aligned}
\partial_{j}^{2}\left(\Gamma_{i} f\right)(x)=\left(\partial_{i j}^{2} f\right)^{2}(x)+\partial_{i} f(x) \partial_{i j j}^{3} f(x)+\frac{k_{i}}{2 x_{i}^{2}}\left(\partial_{j} f(x)-\partial_{j} f\left(\sigma_{i} x\right)\right)^{2}+ \\
\frac{k_{i}}{2 x_{i}^{2}}\left(f(x)-f\left(\sigma_{i} x\right)\right)\left(\partial_{j}^{2} f(x)-\partial_{j}^{2} f\left(\sigma_{i} x\right)\right) .
\end{aligned}
$$


Again, considering (2.11) with $\sigma_{j} x$ instead of $x$ we have that

$$
\begin{aligned}
\frac{k_{j}}{2 x_{j}^{2}}\left(\Gamma_{i} f(x)-\Gamma_{i} f\left(\sigma_{j} x\right)\right) & = \\
& \frac{k_{j}}{4 x_{j}^{2}}\left(\left(\partial_{i} f\right)^{2}(x)-\left(\partial_{i} f\right)^{2}\left(\sigma_{j} x\right)\right)+\frac{k_{j} k_{i}}{8 x_{j}^{2} x_{i}^{2}}\left[\left(f(x)-f\left(\sigma_{i} x\right)\right)^{2}-\left(f\left(\sigma_{j} x\right)-f\left(\sigma_{i} \sigma_{j} x\right)\right)^{2}\right] .
\end{aligned}
$$

Therefore, if $i \neq j$, replacing the equations (2.17), (2.18) and (2.19) in (2.12) we can see that $L_{k}^{j}\left(\Gamma_{i} f\right)$ can be expressed as

$$
\begin{aligned}
& L_{k}^{j}\left(\Gamma_{i} f\right)(x)=\frac{\left(\partial_{i j}^{2} f\right)^{2}(x)}{2}+\frac{\partial_{i} f(x) \partial_{i j j}^{3} f(x)}{2}-x_{j} \partial_{j} f(x) \partial_{i j}^{2} f(x)+\frac{k_{i}}{4 x_{i}^{2}}\left(\partial_{j} f(x)-\partial_{j} f\left(\sigma_{i} x\right)\right)^{2} \\
& +\frac{k_{i}}{4 x_{i}^{2}}\left(f(x)-f\left(\sigma_{i} x\right)\right)\left(\partial_{j}^{2} f(x)-\partial_{j}^{2} f\left(\sigma_{i} x\right)\right)+\frac{k_{j}}{x_{j}} \partial_{i} f(x) \partial_{i j}^{2} f(x) \\
& +\left[\frac{k_{i} k_{j}}{2 x_{i}^{2} x_{j}}-\frac{k_{i} x_{j}}{2 x_{i}^{2}}\right]\left(f(x)-f\left(\sigma_{i} x\right)\right)\left(\partial_{j} f(x)-\partial_{j} f\left(\sigma_{i} x\right)\right)-\frac{k_{j}}{4 x_{j}^{2}}\left(\left(\partial_{i} f\right)^{2}(x)-\left(\partial_{i} f\right)^{2}\left(\sigma_{j} x\right)\right) \\
& -\frac{k_{i} k_{j}}{8 x_{i}^{2} x_{j}^{2}}\left[\left(f(x)-f\left(\sigma_{i} x\right)\right)^{2}-\left(f\left(\sigma_{j} x\right)-f\left(\sigma_{i} \sigma_{j} x\right)\right)^{2}\right] .
\end{aligned}
$$

Thus, taking the sum with respect to $i$ and $j$ in (2.16) and (2.20) we obtain explicitly that

$$
\begin{aligned}
& L_{k}(\Gamma f)(x)=\sum_{i=1}^{d}\left\{\frac{\left(\partial_{i}^{2} f\right)^{2}(x)}{2}+\frac{\partial_{i} f(x) \partial_{i}^{3} f(x)}{2}-x_{i} \partial_{i}^{2} f(x) \partial_{i} f(x)+\frac{k_{i}}{4 x_{i}^{2}}\left(\partial_{i} f(x)+\partial_{i} f\left(\sigma_{i} x\right)\right)^{2}\right. \\
& +\frac{k_{i}}{4 x_{i}^{2}}\left(f(x)-f\left(\sigma_{i} x\right)\right)\left(\partial_{i}^{2} f(x)-\partial_{i}^{2} f\left(\sigma_{i} x\right)\right)-\frac{k_{i}}{4 x_{i}^{2}}\left(\left(\partial_{i} f\right)^{2}(x)-\left(\partial_{i} f\right)^{2}\left(\sigma_{i} x\right)\right) \\
& +\left[\frac{k_{i}^{2}}{2 x_{i}^{3}}-\frac{k_{i}}{x_{i}^{3}}-\frac{k_{i}}{2 x_{i}}\right]\left(f(x)-f\left(\sigma_{i} x\right)\right)\left(\partial_{i} f(x)+\partial_{i} f\left(\sigma_{i} x\right)\right)+\frac{k_{i}}{x_{i}} \partial_{i}^{2} f(x) \partial_{i} f(x) \\
& \left.+\left[\frac{3 k_{i}}{4 x_{i}^{4}}-\frac{k_{i}^{2}}{2 x_{i}^{4}}+\frac{k_{i}}{2 x_{i}^{2}}\right]\left(f(x)-f\left(\sigma_{i} x\right)\right)^{2}\right\}+ \\
& \sum_{i=1}^{d} \sum_{j=1, j \neq i}^{d}\left\{\frac{\left(\partial_{i j}^{2} f\right)^{2}(x)}{2}+\frac{\partial_{i} f(x) \partial_{i j j}^{3} f(x)}{2}-x_{j} \partial_{j} f(x) \partial_{i j}^{2} f(x)+\frac{k_{i}}{4 x_{i}^{2}}\left(\partial_{j} f(x)-\partial_{j} f\left(\sigma_{i} x\right)\right)^{2}\right. \\
& +\frac{k_{i}}{4 x_{i}^{2}}\left(f(x)-f\left(\sigma_{i} x\right)\right)\left(\partial_{j}^{2} f(x)-\partial_{j}^{2} f\left(\sigma_{i} x\right)\right)+\frac{k_{j}}{x_{j}} \partial_{i} f(x) \partial_{i j}^{2} f(x) \\
& +\left[\frac{k_{i} k_{j}}{2 x_{i}^{2} x_{j}}-\frac{k_{i} x_{j}}{2 x_{i}^{2}}\right]\left(f(x)-f\left(\sigma_{i} x\right)\right)\left(\partial_{j} f(x)-\partial_{j} f\left(\sigma_{i} x\right)\right)-\frac{k_{j}}{4 x_{j}^{2}}\left(\left(\partial_{i} f\right)^{2}(x)-\left(\partial_{i} f\right)^{2}\left(\sigma_{j} x\right)\right) \\
& \left.-\frac{k_{i} k_{j}}{8 x_{i}^{2} x_{j}^{2}}\left[\left(f(x)-f\left(\sigma_{i} x\right)\right)^{2}-\left(f\left(\sigma_{j} x\right)-f\left(\sigma_{i} \sigma_{j} x\right)\right)^{2}\right]\right\} \text {. }
\end{aligned}
$$

Now, we will develop the second part of the proof of this proposition calculating the operator 
$\left\langle\nabla f, \nabla \mathrm{L}_{k} \mathrm{f}\right\rangle$, such can be written as

$$
\begin{aligned}
\left\langle\nabla f(x), \nabla L_{k} f(x)\right\rangle & =\sum_{i=1}^{d} \partial_{i} f(x) \partial_{i}\left(L_{k} f\right)(x) \\
& =\sum_{i=1}^{d} \sum_{j=1}^{d} \partial_{i} f(x) \partial_{i}\left(L_{k}^{j} f\right)(x) \\
& =\sum_{i=1}^{d} \partial_{i} f(x) \partial_{i}\left(L_{k}^{i} f\right)(x)+\sum_{i=1}^{d} \sum_{j=1, j \neq i}^{d} \partial_{i} f(x) \partial_{i}\left(L_{k}^{j} f\right)(x) .
\end{aligned}
$$

Again, we consider $i=j$ and $i \neq j$. If $i=j$, from (2.4) we obtain that

$$
\begin{aligned}
& \partial_{i}\left(L_{k}^{i} f\right)(x)= \\
& \frac{\partial_{i}^{3} f(x)}{2}-\partial_{i} f(x)-x_{i} \partial_{i}^{2} f(x)+\frac{k_{i}}{x_{i}} \partial_{i}^{2} f(x)-\frac{k_{i}}{x_{i}^{2}} \partial_{i} f(x)-\frac{k_{i}}{2 x_{i}^{2}}\left(\partial_{i} f(x)+\partial_{i} f\left(\sigma_{i} x\right)\right) \\
& +\frac{k_{i}}{x_{i}^{3}}\left(f(x)-f\left(\sigma_{i} x\right)\right) .
\end{aligned}
$$

But, if $i \neq j$, we get

$$
\partial_{i}\left(L_{k}^{j} f\right)(x)=\frac{\partial_{j j i}^{3} f(x)}{2}+\frac{k_{j}}{x_{j}} \partial_{j i}^{2} f(x)-\frac{k_{j}}{2 x_{j}^{2}}\left(\partial_{i} f(x)-\partial_{i} f\left(\sigma_{j} x\right)\right)-x_{j} \partial_{j i}^{2} f(x) .
$$

Thus, taking the sum with respect to $i$ and $j$ in $(2.22)$ and $(2.23)$ we express

$$
\begin{aligned}
& \left\langle\nabla f(x), \nabla L_{k} f(x)\right\rangle= \\
& \sum_{i=1}^{d}\left\{\frac{\partial_{i}^{3} f(x) \partial_{i} f(x)}{2}-\left(\partial_{i} f\right)^{2}(x)-x_{i} \partial_{i}^{2} f(x) \partial_{i} f(x)+\frac{k_{i}}{x_{i}} \partial_{i}^{2} f(x) \partial_{i} f(x)-\frac{k_{i}}{x_{i}^{2}}\left(\partial_{i} f\right)^{2}(x)\right. \\
& \left.-\frac{k_{i}}{2 x_{i}^{2}}\left(\partial_{i} f(x)+\partial_{i} f\left(\sigma_{i} x\right)\right) \partial_{i} f(x)+\frac{k_{i}}{x_{i}^{3}}\left(f(x)-f\left(\sigma_{i} x\right)\right) \partial_{i} f(x)\right\}+\sum_{i=1}^{d} \sum_{j=1, j \neq i}^{d}\left\{\frac{\partial_{j j i}^{3} f(x) \partial_{i} f(x)}{2}\right. \\
& \left.+\frac{k_{j}}{x_{j}} \partial_{j i}^{2} f(x) \partial_{i} f(x)-\frac{k_{j}}{2 x_{j}^{2}}\left(\partial_{i} f(x)-\partial_{i} f\left(\sigma_{j} x\right)\right) \partial_{i} f(x)-x_{j} \partial_{j i}^{2} f(x) \partial_{i} f(x)\right\} .
\end{aligned}
$$

Finally as third step, we turn now to compute explicitly the terms of the expression

$$
\sum_{i=1}^{d} \sum_{j=1}^{d} \frac{k_{i}}{2 x_{i}^{2}}\left(f(x)-f\left(\sigma_{i} x\right)\right)\left(L_{k}^{j} f(x)-L_{k}^{j} f\left(\sigma_{i} x\right)\right)
$$

Then, if $i=j$, by using (2.4) with $\sigma_{i} x$ instead of $x$, we can write

$$
L_{k}^{i} f\left(\sigma_{i} x\right)=\frac{\partial_{i}^{2} f\left(\sigma_{i} x\right)}{2}-\frac{k_{i}}{x_{i}} \partial_{i} f\left(\sigma_{i} x\right)-\frac{k_{i}}{2 x_{i}^{2}}\left(f\left(\sigma_{i} x\right)-f(x)\right)+x_{i} \partial_{i} f\left(\sigma_{i} x\right),
$$


(remind that $\sigma_{i} x=\left(x_{1}, \ldots,-x_{i}, \ldots, x_{d}\right)$ and $\left.\sigma_{i}\left(\sigma_{i} x\right)=x\right)$, therefore,

$$
\begin{aligned}
& L_{k}^{i} f(x)-L_{k}^{i} f\left(\sigma_{i} x\right)= \\
& \frac{\left(\partial_{i}^{2} f(x)-\partial_{i}^{2} f\left(\sigma_{i} x\right)\right)}{2}+\frac{k_{i}}{x_{i}}\left(\partial_{i} f(x)+\partial_{i} f\left(\sigma_{i} x\right)\right)-\frac{k_{i}}{x_{i}^{2}}\left(f(x)-f\left(\sigma_{i} x\right)\right) \\
& -x_{i}\left(\partial_{i} f(x)+\partial_{i} f\left(\sigma_{i} x\right)\right) .
\end{aligned}
$$

But, if $i \neq j$, we get

$$
\begin{aligned}
& L_{k}^{j} f(x)-L_{k}^{j} f\left(\sigma_{i} x\right)= \\
& \frac{\left(\partial_{j}^{2} f(x)-\partial_{j}^{2} f\left(\sigma_{i} x\right)\right)}{2}+\frac{k_{j}}{x_{j}}\left(\partial_{j} f(x)-\partial_{j} f\left(\sigma_{i} x\right)\right)+\frac{k_{j}}{2 x_{j}^{2}}\left(f\left(\sigma_{j} x\right)-f(x)+f\left(\sigma_{i} x\right)-f\left(\sigma_{j} \sigma_{i} x\right)\right) \\
& -x_{j}\left(\partial_{j} f(x)-\partial_{j} f\left(\sigma_{i} x\right)\right) .
\end{aligned}
$$

So, from (2.25) and (2.26) we can conclude that

$$
\begin{aligned}
& \sum_{i=1}^{d} \sum_{j=1}^{d} \frac{k_{i}}{2 x_{i}^{2}}\left(f(x)-f\left(\sigma_{i} x\right)\right)\left(L_{k}^{j} f(x)-L_{k}^{j} f\left(\sigma_{i} x\right)\right)= \\
& \sum_{i=1}^{d}\left\{\frac{k_{i}}{4 x_{i}^{2}}\left(\partial_{i}^{2} f(x)-\partial_{i}^{2} f\left(\sigma_{i} x\right)\right)\left(f(x)-f\left(\sigma_{i} x\right)\right)+\frac{k_{i}^{2}}{2 x_{i}^{3}}\left(\partial_{i} f(x)+\partial_{i} f\left(\sigma_{i} x\right)\right)\left(f(x)-f\left(\sigma_{i} x\right)\right)\right. \\
& \left.-\frac{k_{i}^{2}}{2 x_{i}^{4}}\left(f(x)-f\left(\sigma_{i} x\right)\right)^{2}-\frac{k_{i}}{2 x_{i}}\left(\partial_{i} f(x)+\partial_{i} f\left(\sigma_{i} x\right)\right)\left(f(x)-f\left(\sigma_{i} x\right)\right)\right\}+ \\
& \sum_{i=1}^{d} \sum_{j=1, j \neq i}^{d}\left\{\frac{k_{i}}{4 x_{i}^{2}}\left(\partial_{j}^{2} f(x)-\partial_{j}^{2} f\left(\sigma_{i} x\right)\right)\left(f(x)-f\left(\sigma_{i} x\right)\right)+\frac{k_{i} k_{j}}{2 x_{i}^{2} x_{j}}\left(\partial_{j} f(x)-\partial_{j} f\left(\sigma_{i} x\right)\right)\left(f(x)-f\left(\sigma_{i} x\right)\right)\right. \\
& +\frac{k_{i} k_{j}}{4 x_{i}^{2} x_{j}^{2}}\left(f\left(\sigma_{j} x\right)-f(x)+f\left(\sigma_{i} x\right)-f\left(\sigma_{j} \sigma_{i} x\right)\right)\left(f(x)-f\left(\sigma_{i} x\right)\right) \\
& \left.-\frac{k_{i} x_{j}}{2 x_{i}^{2}}\left(\partial_{j} f(x)-\partial_{j} f\left(\sigma_{i} x\right)\right)\left(f(x)-f\left(\sigma_{i} x\right)\right)\right\} .
\end{aligned}
$$

Then, at this point in our argument, replacing the identities (2.21), (2.24) and (2.27) in (2.10) and simplifying the terms that are equal, we can write

$$
\Gamma_{2}(f)(x)=E_{1}(x)+E_{2}(x)
$$


where we denote by

$$
\begin{aligned}
& E_{1}(x)=\frac{1}{2}\left\{\sum_{i=1}^{d} \frac{\left(\partial_{i}^{2} f\right)^{2}(x)}{2}+\left(\partial_{i} f\right)^{2}(x)+\frac{k_{i}}{4 x_{i}^{2}}\left(\partial_{i} f(x)+\partial_{i} f\left(\sigma_{i} x\right)\right)^{2}\right. \\
& -\frac{k_{i}}{x_{i}^{3}}\left(\partial_{i} f(x)+\partial_{i} f\left(\sigma_{i} x\right)\right)\left(f(x)-f\left(\sigma_{i} x\right)\right)+\left[\frac{3 k_{i}}{4 x_{i}^{4}}+\frac{k_{i}}{2 x_{i}^{2}}\right]\left(f(x)-f\left(\sigma_{i} x\right)\right)^{2} \\
& -\frac{k_{i}}{4 x_{i}^{2}}\left(\left(\partial_{i} f\right)^{2}(x)-\left(\partial_{i} f\right)^{2}\left(\sigma_{i} x\right)\right)+\frac{k_{i}}{x_{i}^{2}}\left(\partial_{i} f\right)^{2}(x)+\frac{k_{i}}{2 x_{i}^{2}}\left(\partial_{i} f(x)+\partial_{i} f\left(\sigma_{i} x\right)\right) \partial_{i} f(x) \\
& \left.-\frac{k_{i}}{x_{i}^{3}}\left(f(x)-f\left(\sigma_{i} x\right)\right) \partial_{i} f(x)\right\}
\end{aligned}
$$

and

$$
\begin{aligned}
& E_{2}(x)=\frac{1}{2}\left\{\sum_{i=1}^{d} \sum_{j=1, j \neq i}^{d} \frac{\left(\partial_{i j}^{2} f\right)^{2}(x)}{2}+\frac{k_{i}}{4 x_{i}^{2}}\left(\partial_{j} f(x)-\partial_{j} f\left(\sigma_{i} x\right)\right)^{2}-\frac{k_{j}}{4 x_{j}^{2}}\left[\left(\partial_{i} f\right)^{2}(x)-\left(\partial_{i} f\right)^{2}\left(\sigma_{j} x\right)\right]\right. \\
& -\frac{k_{j} k_{i}}{8 x_{j}^{2} x_{i}^{2}}\left[\left(f(x)-f\left(\sigma_{i} x\right)\right)^{2}-\left(f\left(\sigma_{j} x\right)-f\left(\sigma_{i} \sigma_{j} x\right)\right)^{2}\right]+\frac{k_{j}}{2 x_{j}^{2}}\left(\partial_{i} f(x)-\partial_{i} f\left(\sigma_{j} x\right)\right) \partial_{i} f(x) \\
& \left.-\frac{k_{i} k_{j}}{4 x_{i}^{2} x_{j}^{2}}\left[\left(f\left(\sigma_{j} x\right)-f(x)+f\left(\sigma_{i} x\right)-f\left(\sigma_{j} \sigma_{i} x\right)\right)\left(f(x)-f\left(\sigma_{i} x\right)\right)\right]\right\} .
\end{aligned}
$$

Therefore, we only need to express $E_{1}(x)$ and $E_{2}(x)$ more easily. First, we consider $E_{1}(x)$ and associating the terms, we see that

$$
\begin{aligned}
& \frac{k_{i}}{4 x_{i}^{2}}\left(\partial_{i} f(x)+\partial_{i} f\left(\sigma_{i} x\right)\right)^{2}+\frac{k_{i}}{2 x_{i}^{2}}\left(\partial_{i} f(x)+\partial_{i} f\left(\sigma_{i} x\right)\right) \partial_{i} f(x)-\frac{k_{i}}{4 x_{i}^{2}}\left(\left(\partial_{i} f\right)^{2}(x)-\left(\partial_{i} f\right)^{2}\left(\sigma_{i} x\right)\right)= \\
& \frac{k_{i}}{2 x_{i}^{2}}\left(\partial_{i} f(x)+\partial_{i} f\left(\sigma_{i} x\right)\right)^{2} .
\end{aligned}
$$

Now, taking the identity (2.28) and completing squares in $E_{1}(x)$ we obtain that

$$
\begin{aligned}
& \frac{k_{i}}{2 x_{i}^{2}}\left(\partial_{i} f(x)+\partial_{i} f\left(\sigma_{i} x\right)\right)^{2}-\frac{k_{i}}{x_{i}^{3}}\left(\partial_{i} f(x)+\partial_{i} f\left(\sigma_{i} x\right)\right)\left(f(x)-f\left(\sigma_{i} x\right)\right)= \\
& \frac{k_{i}}{2}\left[\frac{\left(\partial_{i} f(x)+\partial_{i} f\left(\sigma_{i} x\right)\right)^{2}}{x_{i}^{2}}-2 \frac{\left(\partial_{i} f(x)+\partial_{i} f\left(\sigma_{i} x\right)\right)}{x_{i}} \frac{\left(f(x)-f\left(\sigma_{i} x\right)\right)}{x_{i}^{2}} \pm \frac{\left(f(x)-f\left(\sigma_{i} x\right)\right)^{2}}{x_{i}^{4}}\right]= \\
& \frac{k_{i}}{2}\left[\left(\frac{\partial_{i} f(x)+\partial_{i} f\left(\sigma_{i} x\right)}{x_{i}}\right)-\left(\frac{f(x)-f\left(\sigma_{i} x\right)}{x_{i}^{2}}\right)\right]^{2}-\frac{k_{i}}{2 x_{i}^{4}}\left(f(x)-f\left(\sigma_{i} x\right)\right)^{2} .
\end{aligned}
$$

This way, from (2.29) we can write

$$
\begin{aligned}
& E_{1}(x)=\frac{1}{2}\left\{\sum_{i=1}^{d} \frac{\left(\partial_{i}^{2} f\right)^{2}(x)}{2}+\left(\partial_{i} f\right)^{2}(x)+\frac{k_{i}}{2}\left[\left(\frac{\partial_{i} f(x)+\partial_{i} f\left(\sigma_{i} x\right)}{x_{i}}\right)-\left(\frac{f(x)-f\left(\sigma_{i} x\right)}{x_{i}^{2}}\right)\right]^{2}\right. \\
& \left.+\left[\frac{k_{i}}{4 x_{i}^{4}}+\frac{k_{i}}{2 x_{i}^{2}}\right]\left(f(x)-f\left(\sigma_{i} x\right)\right)^{2}+\frac{k_{i}}{x_{i}^{2}}\left(\partial_{i} f\right)^{2}(x)-\frac{k_{i}}{x_{i}^{3}}\left(f(x)-f\left(\sigma_{i} x\right)\right) \partial_{i} f(x)\right\}
\end{aligned}
$$


since, $\left(3 k_{i} / 4 x_{i}^{4}\right)-\left(k_{i} / 2 x_{i}^{4}\right)=k_{i} / 4 x_{i}^{4}$.

Then, associating the terms in the above expression, we have

$$
\frac{k_{i}}{x_{i}^{2}}\left(\partial_{i} f\right)^{2}(x)-\frac{k_{i}}{x_{i}^{3}}\left(f(x)-f\left(\sigma_{i} x\right)\right) \partial_{i} f(x)+\frac{k_{i}}{4 x_{i}^{4}}\left(f(x)-f\left(\sigma_{i} x\right)\right)^{2}=k_{i}\left[\frac{\partial_{i} f(x)}{x_{i}}-\left(\frac{f(x)-f\left(\sigma_{i} x\right)}{2 x_{i}^{2}}\right)\right]^{2}
$$

and therefore, we can conclude that

$$
\begin{aligned}
E_{1}(x)= & \frac{1}{2}\left\{\sum_{i=1}^{d} \frac{\left(\partial_{i}^{2} f\right)^{2}(x)}{2}+\left(\partial_{i} f\right)^{2}(x)+\frac{k_{i}}{2}\left[\left(\frac{\partial_{i} f(x)+\partial_{i} f\left(\sigma_{i} x\right)}{x_{i}}\right)-\left(\frac{f(x)-f\left(\sigma_{i} x\right)}{x_{i}^{2}}\right)\right]^{2}\right. \\
& \left.+k_{i}\left[\frac{\partial_{i} f(x)}{x_{i}}-\left(\frac{f(x)-f\left(\sigma_{i} x\right)}{2 x_{i}^{2}}\right)\right]^{2}+\frac{k_{i}}{2 x_{i}^{2}}\left(f(x)-f\left(\sigma_{i} x\right)\right)^{2}\right\} .
\end{aligned}
$$

Now, we consider $E_{2}(x)$. Once more, we observe that

$$
-\frac{k_{j}}{4 x_{j}^{2}}\left[\left(\partial_{i} f\right)^{2}(x)-\left(\partial_{i} f\right)^{2}\left(\sigma_{j} x\right)\right]+\frac{k_{j}}{2 x_{j}^{2}}\left(\partial_{i} f(x)-\partial_{i} f\left(\sigma_{j} x\right)\right) \partial_{i} f(x)=\frac{k_{j}}{4 x_{j}^{2}}\left[\partial_{i} f(x)-\partial_{i} f\left(\sigma_{j} x\right)\right]^{2} .
$$

Moreover, associating the terms

$$
\begin{aligned}
& -\frac{k_{j} k_{i}}{4 x_{j}^{2} x_{i}^{2}}\left(f\left(\sigma_{j} x\right)-f(x)+f\left(\sigma_{i} x\right)-f\left(\sigma_{j} \sigma_{i} x\right)\right)\left(f(x)-f\left(\sigma_{i} x\right)\right)= \\
& \frac{k_{j} k_{i}}{8 x_{j}^{2} x_{i}^{2}}\left[2\left(f(x)-f\left(\sigma_{i} x\right)\right)^{2}-2\left(f\left(\sigma_{j} x\right)-f\left(\sigma_{j} \sigma_{i} x\right)\right)\left(f(x)-f\left(\sigma_{i} x\right)\right)\right],
\end{aligned}
$$

then

$$
\begin{gathered}
-\frac{k_{j} k_{i}}{8 x_{j}^{2} x_{i}^{2}}\left[\left(f(x)-f\left(\sigma_{i} x\right)\right)^{2}-\left(f\left(\sigma_{j} x\right)-f\left(\sigma_{i} \sigma_{j} x\right)\right)^{2}\right]+ \\
\frac{k_{j} k_{i}}{8 x_{j}^{2} x_{i}^{2}}\left[2\left(f(x)-f\left(\sigma_{i} x\right)\right)^{2}-2\left(f\left(\sigma_{j} x\right)-f\left(\sigma_{j} \sigma_{i} x\right)\right)\left(f(x)-f\left(\sigma_{i} x\right)\right)\right]= \\
\frac{k_{j} k_{i}}{8 x_{j}^{2} x_{i}^{2}}\left[\left(f(x)-f\left(\sigma_{i} x\right)\right)-\left(f\left(\sigma_{j} x\right)-f\left(\sigma_{i} \sigma_{j} x\right)\right)\right]^{2} .
\end{gathered}
$$

Therefore, replacing (2.31) and (2.32) in $\mathrm{E}_{2}(\mathrm{x})$, we express

$$
\begin{aligned}
E_{2}(x)= & \frac{1}{2}\left\{\sum_{i=1}^{d} \sum_{j=1, j \neq i}^{d} \frac{\left(\partial_{i j}^{2} f\right)^{2}(x)}{2}+\frac{k_{i}}{4 x_{i}^{2}}\left[\partial_{j} f(x)-\partial_{j} f\left(\sigma_{i} x\right)\right]^{2}+\right. \\
& \left.\frac{k_{j}}{4 x_{j}^{2}}\left[\partial_{i} f(x)-\partial_{i} f\left(\sigma_{j} x\right)\right]^{2}+\frac{k_{j} k_{i}}{8 x_{j}^{2} x_{i}^{2}}\left[\left(f(x)-f\left(\sigma_{i} x\right)\right)-\left(f\left(\sigma_{j} x\right)-f\left(\sigma_{i} \sigma_{j} x\right)\right)\right]^{2}\right\}
\end{aligned}
$$

and finally, the sum of (2.30) and (2.33) allows us to obtain the result of the Proposition.

In consecuense, we are able to prove that the Dunkl-Ornstein-Uhlenbeck differential operator, $L_{k}$, defined as in (1.2) and associated with the $\mathbb{Z}_{2}^{\mathrm{d}}$ group, satisfies a $\operatorname{CD}(\rho, \infty)$-inequality, if $0 \leq \rho \leq 1$ 
Theorem 2.4. Let $\mathrm{L}_{\mathrm{k}}$ be the Dunkl-Ornstein-Uhlenbeck differential operator defined as in (1.2). Then, if $0 \leq \rho \leq 1$, the $\mathrm{CD}(\rho, \infty)$-inequality is satisfied.

Proof. From Lemma 2.1 and the Proposition 2.3, we have that $\Gamma_{2}(f)(x) \geq \rho \Gamma(f)(x)$ is true, if and only if,

$$
\begin{aligned}
& \sum_{i=1}^{d}\left\{\frac{\left(\partial_{i}^{2} f\right)^{2}(x)}{4}+(1-\rho) \frac{\left(\partial_{i} f\right)^{2}(x)}{2}+\frac{k_{i}}{4}\left[\frac{\left(\partial_{i} f(x)+\partial_{i} f\left(\sigma_{i} x\right)\right)}{x_{i}}-\frac{\left(f(x)-f\left(\sigma_{i} x\right)\right)}{x_{i}^{2}}\right]^{2}\right. \\
& \left.+\frac{k_{i}}{2}\left[\frac{\partial_{i} f(x)}{x_{i}}-\frac{\left(f(x)-f\left(\sigma_{i} x\right)\right)}{2 x_{i}^{2}}\right]^{2}+(1-\rho) \frac{k_{i}}{4 x_{i}^{2}}\left(f(x)-f\left(\sigma_{i} x\right)\right)^{2}\right\} \\
& +\sum_{i=1}^{d} \sum_{j=1, j \neq i}^{d}\left\{\frac{\left(\partial_{i j}^{2} f\right)^{2}(x)}{4}+\frac{k_{i}}{8 x_{i}^{2}}\left(\partial_{j} f(x)-\partial_{j} f\left(\sigma_{i} x\right)\right)^{2}+\frac{k_{j}}{8 x_{j}^{2}}\left(\partial_{i} f(x)-\partial_{i} f\left(\sigma_{j} x\right)\right)^{2}\right. \\
& \left.+\frac{k_{i} k_{j}}{16 x_{i}^{2} x_{j}^{2}}\left[\left(f(x)-f\left(\sigma_{i} x\right)\right)-\left(f\left(\sigma_{j} x\right)-f\left(\sigma_{i} \sigma_{j} x\right)\right)\right]^{2}\right\} \geq 0 .
\end{aligned}
$$

Then, we only need to choose $0 \leq \rho \leq 1$ to obtain the result.

Now, again we consider the family of measures $M_{t}^{k}(x, d y)$ defined in (1.5). If the measures $m_{k}$ are replaced by $M_{\mathfrak{t}}^{k}(x, d y)$, then the logarithmic Sobolev inequalities $\operatorname{LS}(A, C)$ can be rewritten as

$$
O_{t}^{k}\left(f^{2} \log f^{2}\right)-O_{t}^{k}\left(f^{2}\right) \log O_{t}^{k}\left(f^{2}\right) \leq A(t) O_{t}^{k}\left(f^{2}\right)+c(t) O_{t}^{k}(\Gamma f)
$$

and if $A=0$,

$$
O_{t}^{k}\left(f^{2} \log f^{2}\right)-O_{t}^{k}\left(f^{2}\right) \log O_{t}^{k}\left(f^{2}\right) \leq c(t) O_{t}^{k}(\Gamma f),
$$

which are known as local Log-Sobolev inequalities and local tight-Log-Sobolev inequalities respectively, (see [2]). Therefore, from the general criterion of D. Bakry and M. Emery cf. [1] we have that the curvature inequality $\mathrm{C}(\rho, \infty)$ is equivalent to the local tight-Log-Sobolev inequality with $c(t)=\frac{1-e^{-2 \rho t}}{\rho}$, (for details, we refer the reader to [2, Proposition 2.6]). Thus, from Corolario 2.4 we obtain that inequality (2.34) is true and therefore,

$$
\begin{aligned}
\int_{\mathbb{R}^{d}} O_{t}^{k}\left(f^{2} \log f^{2}\right)(x) m_{k}(d x)-\int_{\mathbb{R}^{d}} O_{t}^{k}\left(f^{2}\right)(x) \log O_{t}^{k}\left(f^{2}\right)(x) m_{k}(d x) & \\
& \leq c(t) \int_{\mathbb{R}^{d}} O_{t}^{k}(\Gamma f)(x) m_{k}(d x),
\end{aligned}
$$

where $\Gamma$ is defined as in the Lemma 2.1 Then, by using the propertie

$$
\int_{\mathbb{R}^{d}} O_{t}^{k} f(x) m_{k}(d x)=\int_{\mathbb{R}^{d}} f(x) m_{k}(d x),
$$

we can conclude that

$$
\operatorname{Ent}\left(\mathrm{f}^{2}\right) \leq \mathrm{CE}(\mathrm{f}), \quad \forall \mathrm{f} \in \mathcal{A} .
$$


In a general context, L. Gross [8, 7] proved that Logarithmic Sobolev inequality is equivalent to the fact that for any $t>0$ and $p \in(1, \infty)$,

$$
\left\|H_{t} f\right\|_{q(t)} \leq e^{m(t)}\|f\|_{p}
$$

where $\left(\mathrm{H}_{\mathrm{t}}\right)_{\mathrm{t}}$ is a diffusion semigroup or a symmetric Markov semigroup and the functions $\mathrm{q}(\mathrm{t})$ and $m(t)$ are defined by

$$
\frac{q(t)-1}{p-1}=\exp (4 t / C) \text { and } m(t)=\frac{A}{16}\left(\frac{1}{p}-\frac{1}{q(t)}\right)
$$

(see [8, Theorems 1 and 2]).

Particularly, tight-Logarithmic-Sobolev inequality is equivalent to the hypercontractivity property. In consecuense, we can conclude that the Dunkl-Ornstein-Uhlenbeck semigroup, $\left\{\mathrm{O}_{t}^{k}\right\}_{t \geq 0}$, is hypercontractive, $\forall t>0$ and $1<p<\infty$. This means,

$$
\left\|\mathrm{O}_{\mathrm{t}}^{\mathrm{k}} \mathrm{f}\right\|_{\mathrm{q}(\mathrm{t}), \mathrm{m}_{\mathrm{k}}} \leq\|\mathrm{f}\|_{\mathrm{p}, \mathrm{m}_{\mathrm{k}}}, \quad \forall \mathrm{f} \in \mathrm{L}^{\mathrm{p}}\left(\mathrm{m}_{\mathrm{k}}\right)
$$

where, $\exp (4 t / C)=(q(t)-1) /(p-1)$ for some positive constant $C$.

By using subordination formula we obtain the same result for the Dunkl-Poisson semigroup $\left\{P_{t}^{k}\right\}_{t} \geq 0$.

\subsection{Applications}

As a consequence of the hypercontractivity propertie of $\left\{\mathrm{O}_{t}^{k}\right\}_{t \geq 0}$ semigroup, we obtain the $\mathrm{L}^{p}\left(\mathrm{~m}_{k}\right)$ continuity of $J_{\mathfrak{n}}^{k}$ operators for every $1<p<\infty$ and $n=0,1,2, \ldots$ The reasoning is similar as in the case of classical Ornstein-Uhlenbeck semigroup and we refer the reader to [17, Lemma 1.1], where the identity $\mathrm{O}_{\mathfrak{t}}^{k}\left(J_{\mathfrak{n}}^{k} \mathrm{f}\right)=e^{-\mathfrak{n t}} J_{\mathfrak{n}}^{k} \mathrm{f}$, if $\mathrm{f} \in \mathcal{A}$, is a key condition in the argument. Moreover, for $1<p<\infty$ and $n \in \mathbb{N}$, there exist a constant $C_{p, n}>0$, such that,

$$
\left\|\mathrm{O}_{\mathrm{t}}^{\mathrm{k}}\left(\mathrm{I}-\cdots-\mathrm{J}_{\mathrm{n}-1}^{\mathrm{k}}\right) \mathrm{f}\right\|_{p, m_{k}} \leq \mathrm{C}_{\mathrm{p}, \mathrm{n}} \mathrm{e}^{-\mathrm{nt}}\|\mathrm{f}\|_{p, m_{k}}
$$

and since the development is similar to the classical Ornstein-Uhlenbeck semigroup, we omit the details and refer to [17, Lemma 1.2]. Then we extend the celebrated P.A Meyer's multiplier theorem to Dunkl-Ornstein-Uhlenbeck semigroup and the $\mathbb{Z}_{2}^{\mathrm{d}}$ group. A first version of this theorem, associated with Hermite expansions, has be obtained in [17, Theorem 1.1] (see also, [18]). Afterwards, similar versions to Laguerre and Jacobi setting have been obtained in [6. Theorem 3.4] and [10, Theorem 4.1], respectively.

Theorem 2.5 (Meyer's multiplier theorem). Let $\left\{\mathrm{O}_{\mathrm{t}}^{k}\right\}_{\mathrm{t} \geq 0}$ be the Dunkl-Onstein-Uhlenbeck semigroup. Assume that $\mathrm{h}$ is a function, which is analytic in a neighborhood of the origin. Let $\{\psi(\mathfrak{n})\}_{\mathfrak{n} \in \mathbb{N}}$ 
be a sequence of real numbers, such that $\psi(\mathrm{n})=\mathrm{h}\left(\mathrm{n}^{-\beta}\right), \forall \mathrm{n} \geq \mathrm{n}_{0}$ and some $\beta \in(0,1]$. Then, the operator

$$
T_{\psi} f=\sum_{n \geq 0} \psi(n) J_{n}^{k} f, \quad f=\sum_{n \geq 0} J_{n}^{k} f
$$

defined initially in $\mathrm{L}^{2}\left(\mathrm{~m}_{\mathrm{k}}\right)$, has a unique continuous linear extension to each of the spaces $\mathrm{L}^{\mathrm{p}}\left(\mathrm{m}_{\mathrm{k}}\right)$, for $1<p<\infty$.

Next, we consider the fractional integrals, the fractional derivatives and the Bessel potentials associated to the differential operator $L_{k}$ and the $\mathbb{Z}_{2}^{d}$ group. Since, $L_{k}$ is symmetric and has a selfadjoint extension, these can be defined by standarts ways, by example by spectral representation of Bochner subordination. However, the use of these fractional operators together with Meyer's multipliers theorem allows us to obtain a characterization of Dunkl-potential spaces, similar to the classic case (see [15]).

Then, Dunkl-fractional integral of order $s>0$, associated to Dunkl-Ornstein-Uhlenbeck differential operator and the $\mathbb{Z}_{2}^{\mathrm{d}}$ group, is defined by

$$
\mathrm{I}_{\mathrm{k}}^{\mathrm{s}}=\left(-\mathrm{L}_{\mathrm{k}}\right)^{-\mathrm{s} / 2} \Pi_{0}
$$

where $\Pi_{0}$ denotes the orthogonal projection onto the orthogonal complement of the subspace spanned by the constant functions. Immediately, from (1.3) we have

$$
\mathrm{I}_{\mathrm{k}}^{\mathrm{s}}\left(\mathrm{h}_{v}^{\mathrm{k}}\right)=|v|^{-\mathrm{s} / 2} \mathrm{~h}_{v}^{\mathrm{k}}, \quad|v|>0, \quad f \in \mathcal{A},
$$

and an integral representation of $\mathrm{I}_{\mathrm{k}}^{\mathrm{s}} \mathrm{f}$ can be obtained

$$
I_{k}^{s} f=\frac{1}{\Gamma(s)} \int_{0}^{\infty} t^{s-1} P_{t}^{k}\left(I-J_{0}^{k}\right) f d t
$$

which makes sense, for all $f \in L^{p}\left(m_{k}\right)$, by means of (2.35) and subordination formula, because

$$
\left\|I_{k}^{s} f\right\|_{p, m_{k}} \leq A_{p}\|f\|_{p, m_{k}} \text { for } s>0, \text { and } 1<p<\infty
$$

(we refer the reader to [6, 9] and [10]).

Also, we introduce the fractional derivative in the $\mathbb{Z}_{2}^{\mathrm{d}}$-Dunkl setting which is given formally by

$$
\mathrm{D}_{\mathrm{k}}^{\mathrm{s}}=\left(-\mathrm{L}_{\mathrm{k}}\right)^{\mathrm{s} / 2}
$$

For the generalized Hermite polynomials we have

$$
\mathrm{D}_{\mathrm{k}}^{\mathrm{s}}\left(\mathrm{h}_{v}^{\mathrm{k}}\right)=|v|^{\mathrm{s} / 2} \mathrm{~h}_{v}^{\mathrm{k}}, \quad \forall \mathrm{s}>0
$$

and therefore, by using the density of polynomials in $L^{p}\left(m_{k}\right)$, the derivative $D_{k}^{s}$ can be extended to $\mathrm{L}^{\mathrm{p}}\left(\mathrm{m}_{\mathrm{k}}\right)$. Particularly, if $0<s<1$, we can write

$$
D_{k}^{s} f=\frac{1}{C_{s}} \int_{0}^{\infty} t^{s-1}\left(P_{t}^{k} f-f\right) d t, \quad \text { where, } \quad C_{s}=\int_{0}^{\infty} u^{-s-1}\left(e^{-u}-1\right) d u .
$$


The identity (2.37) may be regarded as the definiton of $D_{k}^{s}$, with $0<s<1$, for all $f \in C_{B}^{2}\left(\mathbb{R}^{d}\right)$, or for all $f$ for which the corresponding integral is absolutely convergent. Moreover, if $f$ is a polynomial, we get

$$
D_{k}^{s}\left(I_{k}^{s} f\right)=I_{k}^{s}\left(D_{k}^{s} f\right)=\Pi_{0} f
$$

Now, the Dunkl-Bessel potential operator, associated to the Dunkl-Ornstein-Uhlenbeck differential operator and the $\mathbb{Z}_{2}^{\mathrm{d}}$ group, is defined as

$$
\left(I-L_{k}\right)^{-s / 2} f=\sum_{n=0}^{\infty}(1+n)^{-s / 2} J_{n}^{k} f, \quad f \in \mathcal{A}
$$

and we defined the Dunkl-potential spaces $L_{k}^{p, s}\left(m_{k}\right)$, associated with generalized Hermite expansions, as the completion of the space of all polynomials with respect to the norm

$$
\|f\|_{p, s}=\left\|\left(I-L_{k}\right)^{s / 2} f\right\|_{p, m_{k}} .
$$

By means of Meyer's multiplier theorem, we can observe that the Dunkl-Bessel potential operator extends to a continuous linear operator on $L^{p}\left(m_{k}\right)$, (for a similar argument see e.g Lemma 6.1 in [6]). Also, the potential spaces have the following properties:

i) If $1 \leq p \leq q$, then $L_{k}^{q, s}\left(m_{k}\right) \subset L_{k}^{p, s}\left(m_{k}\right)$, for each $s \geq 0$.

ii) If $0 \leq s \leq r$, then $L_{k}^{p, r}\left(m_{k}\right) \subset L_{k}^{p, s}\left(m_{k}\right)$, for each $1<p<\infty$.

Moreover, the embeddings in i) and ii) are continuous. Again, we omit the proofs of these two facts, but we refer the reader to the Proposition 2.2 in [9] and the Proposition 6.3 in [6].

Finally, the following theorem allows us to extend the Dunkl-fractional derivative, $\mathrm{D}_{\mathrm{k}}^{\mathrm{s}}$, to the potential spaces $L_{k}^{p, s}\left(m_{k}\right)$, for $1<p<\infty, s>0$ and associated to generalized Hermite expansions, where we consider the $\mathbb{Z}_{2}^{\mathrm{d}}$ group. Thus, the union of these spaces;

$$
L_{k}^{s}\left(m_{k}\right)=\bigcup_{p>1} L_{k}^{p, s}\left(m_{k}\right)
$$

make up a natural domain of $\mathrm{D}_{\mathrm{k}}^{\mathrm{s}}$. Similar versions of this theorem has been obtained in [9, Theorem 2.2], where we consider classical Hermite expansions, in [6, Theorem 6.4] related to Laguerrre expansions and afterwards, in [10, Theorem 5.1] in the Jacobi context.

Theorem 2.6. Let $s \geq 0$ and $1<p<\infty$.

i) If $\left\{P_{n}\right\}_{n}$ is a sequence of polynomials such that $\lim _{n \rightarrow \infty} P_{n}=f$ in $L_{s}^{p}\left(m_{k}\right)$, then $\lim _{n} D_{k}^{s} P_{n}$ exists in $\mathrm{L}_{\mathrm{k}}^{\mathrm{p}, \mathrm{s}}\left(\mathrm{m}_{\mathrm{k}}\right)$ and does not depend on the choice of a sequence $\left\{\mathrm{P}_{\mathrm{n}}\right\}_{n}$.

If $\mathrm{f} \in \mathrm{L}_{\mathrm{k}}^{\mathrm{p}, \mathrm{s}}\left(\mathrm{m}_{\mathrm{k}}\right) \cap \mathrm{L}_{\mathrm{k}}^{\mathrm{p}, \mathrm{r}}\left(\mathrm{m}_{\mathrm{k}}\right)$, then the limit does not depend on the choice of $\mathrm{p}$ or $\mathrm{r}$. Thus, $D_{k}^{s} f=\lim _{n \rightarrow \infty} D_{k}^{s} P_{n}$ in $L_{k}^{s, p}\left(m_{k}\right), \quad \lim _{n \rightarrow \infty} P_{n}=f$ in $L_{k}^{p, s}\left(m_{k}\right)$, $\mathrm{f} \in \mathrm{L}_{\mathrm{k}}^{\mathrm{s}}\left(\mathrm{m}_{\mathrm{k}}\right)$, is well defined. 
ii) $\mathrm{f} \in \mathrm{L}_{\mathrm{k}}^{\mathrm{p}, \mathrm{s}}\left(\mathrm{m}_{\mathrm{k}}\right)$ if and only if $\mathrm{D}_{\mathrm{k}}^{\mathrm{s}} \mathrm{f} \in \mathrm{L}^{\mathrm{p}}\left(\mathrm{m}_{\mathrm{k}}\right)$. Moreover,

$$
B_{p, s}\|f\|_{p, s} \leq\left\|D_{k}^{s} f\right\|_{p, m_{k}} \leq A_{p, s}\|f\|_{p, s} .
$$

\section{References}

[1] D. Bakry, M. Emery, Hypercontractivité de semi-groupes de diffusion, C.R Acad. Sci. Paris Sér, 1299, (15), 775-778, (1984).

[2] D. Bakry, On Sobolev and Logarithmic Sobolev inequalities for Markov semigroups, in: New Trends in stochastic analysis (Charingworth), 43-75, (1994). River Edge N.J 1997. TaniguchiSymposium World. Sci. Publishing.

[3] D. Bakry, Functional inequalities for Markov semigroups, Probability measures on groups: Recent directions and trends, Tata Institute of Fundamental Research, Mumbai, 91-147, (2006).

[4] T.S Chihara, An introduction to orthogonal polynomials, Gordon and Breach, New York, (1978).

[5] C. F. Dunkl, Differential-difference operators associated to reflection groups, Trans. Amer. Math. Soc, 311, 167-183, (1989).

[6] P. Graczky, J. Loeb, I. López, A. Nowak, W. Urbina, Higher order Riesz transforms, fractional derivatives and Sobolev spaces for Laguerre expansions, J. Math. Pures et Appl, 84, 375-405, (2005).

[7] L. Gross, Logarithmic Sobolev inequalities and contractivity properties of semigroups, in: Dirichlet forms (Varenna, 1992) Springer, Verling, 54-88, (1993).

[8] L. Gross, Logarithmic Sobolev inequalities, Amer. J. Math. 97, 1061-1083, (1976).

[9] I. López, W. Urbina, Fractional differentiation for the Gaussian measure and applications, Bull. Sci. Math, 83, Vol 128, Issue 7, 587-603, (2004).

[10] I. López, Operators associated with the Jacobi semigroup, J. Approx. Theory, 161, 385-410, (2009).

[11] M. Rösler, Generalize Hermite polynomials and the heat equation for Dunkl operators, Commun. Math. Phys. 192, 519-542, (1998).

[12] M. Rösler, M. Voit Markov processes related with Dunkl operators, Adv. in Appl. Math, 21, $575-643,(1998)$.

[13] M. Rösler Dunkl operators. Theory and applications, Orthogonal polynomials and special functions, (Leuven 2002), 93-135. Lecture Notes in Math. 1817, Springer-Berlin, (2003). 
[14] M. Rösler, M. Voit, Dunkl theory, convolution algebras and related Markov processes, in: Harmonic and stochastic analysis of Dunkl processes, eds. P. Graczyk, M. Rösler, M. Yor, Travaux en cours 71, 1-112, Hermann, Paris, (2008).

[15] E. Stein, The Characterization of Functions Arising as Potentials I, Bull. Amer. Math. Soc. 97, 102-104, (1961). II (ibid) 68, 577-582, (1962).

[16] E. Stein, Topics in Harmonic Analysis related to the Littlewood-Paley Theory, Princenton Univ. Press. Princenton (1971).

[17] H. Sugita, Sobolev spaces of Wiener functionals and Malliavin's calculus, J. Math. Kyoto Univ, 25-1, 31-48, (1985).

[18] S. Watanabe, M. Gopalan Nair, B. Rajeev Lectures on Stochastic differential equations and Malliavin Calculus, Tata Institute of Fundamental Research, Vol. 73, Springer Verlag, Berlin/Heidelberg/New York/Tokyo, (1984). 